\title{
Long cryopreserved lab-adapted Plasmodium falciparum increases resistance to chloroquine but not its susceptibility
}

\author{
Pua Jing Yit ${ }^{1}$, Nurhidanatasha Abu Bakar ${ }^{1}$, Nik Abdul Aziz Nik Kamarudin ${ }^{2}$, \\ Siti Zulaiha Ghazali ${ }^{2}$ and Khairul Mohd Fadzli Mustaffa ${ }^{2, *}$ \\ ${ }^{1}$ School of Health Sciences, Health Campus, Universiti Sains Malaysia, 16150 Kubang Kerian, \\ Kelantan, Malaysia. \\ ${ }^{2}$ Institute for Research in Molecular Medicine, Health Campus, Universiti Sains Malaysia, 16150 \\ Kubang Kerian, Kelantan, Malaysia.
}

\section{ABSTRACT}

Background: Plasmodium falciparum is a deadly protozoan that is accountable for malaria and chloroquine was the first-line antimalarial drug before its withdrawal and replaced by artemisinin. To date, several studies showed that $P$. falciparum had regained its sensitivity towards chloroquine after its withdrawal for decades. By understanding the basic principle and mechanism of chloroquine resistance in P. falciparum, at the molecular level, it would be valuable prior to the reintroduction of chloroquine as a first-line anti-malarial drug for malaria treatment. Thus, this study was conducted to determine the chloroquine resistance level of long preserved lab-adapted $P$. falciparum strain. Methodology: By using 14 years (2006-2020) cryopreserved chloroquinesensitive (3D7) and chloroquine-resistant (W2) lab-adapted P. falciparum strains, the strains were subjected to continuous culture for three months before in vitro drug susceptibility assay and single nucleotide polymorphisms (SNP) analysis on Pfcrt and Pfmdr-1 gene for both strains. Results: This study shows the IC $\mathrm{C}_{50}$ chloroquine of lab-adapted P. falciparum 3D7 and W2 strains were at 32.98 $\mathrm{nM}$ and $691.21 \mathrm{nM}$, respectively and both strains showed 3 -fold higher IC 50 when compared to their susceptibility before cryopreserved (3D7; 13.84nM and W2;208.27 nM). The SNPs result showed a consistent amino acid substitution at position 76 (K to T) on PfCRT and 86 (N to Y) in Pfmdr-1 gene which concordance with other studies before preservation. Conclusion: Thus, this study shows that long cryopreserved of lab-adapted $P$. falciparum increases the chloroquine resistance

* Correspondence Khairul Mohd Fadzli Mustaffa Institute for Research in Molecular Medicine, Health Campus Universiti Sains Malaysia 16150 Kubang Kerian, Kelantan, Malaysia khairulmf@usm.my Tel: +609-767 2435

Received: 19 July 2020 Revised: 17 September 2020

Accepted: 13 October 2020 Published: 6 November 2020

doi https://doi.org/10.28916/lsmb.4.9.2020.66 level but not exhibited any change in susceptibility.

Keywords: Plasmodium falciparum; chloroquine resistance; SNPs, PfCRT; PfMDR-1

\section{INTRODUCTION}

Malaria is a mosquito-borne infectious disease caused by parasites that are transmitted from human to human or from animal to human via the bites of infected female Anopheles mosquitoes. According to the World Malaria Report 2018 published by the World Health Organization (WHO), an estimated 219 million malaria cases were reported worldwide in 2017. Based on the report, the African region contributed the most to the cases (200 million or 92\% in 2017), followed by Southeast Asia (11 million or 5\%). Although there were an estimated 20 million malaria cases fewer from 2010 to 2017, the period of 2015 to 2017 highlighted that there was no significant progress in eliminating global malaria cases (World Health Organization, 2019). Nevertheless, malaria remains a major global health problem in the $21^{\text {st }}$ century and more than half of the world's population is at the risk of acquiring malaria. 
Malaria in humans is caused by the protozoan parasite of the genus Plasmodium, such as Plasmodium falciparum (P. falciparum), Plasmodium malariae (P. malariae), Plasmodium ovale (P. ovale) and Plasmodium vivax (P. vivax) (Perlmann \& Troye-Blomberg, 2000; Sharma \& Khanduri, 2009; Siswantoro et al., 2011; Sutherland et al., 2010). In 2000, Plasmodium knowlesi (P. knowlesi) was rectified as the fifth human malaria parasite that initially infected monkeys (Sabbatani et al., 2010; Tang et al., 2010).

Among the five human Plasmodium, P. falciparum has extensively been explored as it is the most prevalent malaria parasite in most of the world regions, the most clinically important infection due to its high risk of death and the parasite develop resistance towards chloroquine as its first-line therapy. Other than that, $P$. falciparum is among three human malaria parasites other than P. vivax (Mehlotra et al., 2017) and $P$. knowlesi (Butcher et al., 1971) that could only be continuously cultured to its complete life-cycle in the laboratory. Therefore, cryopreservation is the only procedure to banking the isolates and to have a sustainability supply of the parasite stock for in vitro experiment. Due to high frequency of freezing and thawing, there is a need to characterize the isolates for it variations such as drug-response, and virulent after long storage in liquid nitrogen. Other than that, studies on the genetic characteristics of drug resistance in the isolates provide essential information on the extent to which mutations involved (WHO memorandums, 1981).

There are two genes associated with the chloroquine-resistant in $P$. falciparum, which is Pfcrt (Plasmodium falciparum chloroquine resistance transporter) and Pfmdr-1 (Plasmodium falciparum multiple drug resistance 1) (Cui et al., 2015). The protein encoded from both genes that found at the food vacuole membrane of $P$. falciparum, which is also the site of chloroquine actions (Antony et al., 2016). Although the involvement of PfMDR-1 protein in chloroquine-resistant is not very clear, several studies indicate that the existence of these two markers causes more resistance to chloroquine than just a single marker. SNPs that occur at both genes result in the encoding of different amino acids that cause the protein to be inactive and led to chloroquine resistance. The mutant $\mathrm{Pfcrt}$ alleles found in chloroquine-resistant parasites contain several point mutations, and the pattern of mutations depending on the region where its originated (Sindhu et al. 2002, Basco and Ringwald 2001). Chloroquine-resistant parasite from South East Asia and Africa carry point mutation at codon $74,75,76,220,271,326,356$ and 371 . Meanwhile, South America strain, carry point mutation at position codon 76, 220 or 72, 326 and 356 or 75,97 and 371 . However, a point mutation at codon 76 plays an important role in conferring chloroquine resistant (Basco and Ringwald 2001, Fidock et al. 2000). For Pfmdr-1, a point mutation at codon 86,184,1034, 1042 and 1246 have been proposed to be associated with chloroquine-resistant (Reed et al. 2000). Among those genes, the mutation at codon 86 from asparagine to tyrosine has been reported widely. Thus, this present study is aimed to characterize drug susceptibility of long cryopreserved P. falciparum in our biobank as this may help us inventories and establishing chloroquine drug-baseline standard for future work.

\section{MATERIALS AND METHODS}

Cultivation of malaria parasites

Fourteen years old cryopreserved $P$. falciparum chloroquinesensitive 3D7 (MRA-102; Amsterdam) and chloroquine-resistance W2 (MRA-157; Indochina) strains obtained through BEI Resources, NIAID, $\mathrm{NIH}$ in 2006 were cultured by referring to Frederick L. Schuster's method (2002). Both strains were cultivated continuously in RPMI-1640 media, supplemented with 10\% AlbuMAX (Thermo Fisher Scientific, USA) and $10 \%$ Type $\mathrm{O}^{+}$washed RBCs. Culture flask was maintained in a $\mathrm{CO}_{2}$ incubator at $37 \pm 0.5^{\circ} \mathrm{C}$ under a regulated gas mixture of $\mathrm{N}_{2}$ (90$92 \%), \mathrm{CO}_{2}(5 \%)$ and $\mathrm{O}_{2}(3-5 \%)$ for optimal parasite growth. The culture was continuously maintained at $<10 \%$ parasitaemia in a $2-4 \%$ haematocrit for 3 months and synchronized every 2 weeks with 5\% sorbitol Lambros and Vanderberg, 1979).

\section{In vitro drug susceptibility assay}

Drug susceptibility assay was conducted for detection of $P$. falciparum susceptibility to chloroquine diphosphate (molecular weight of base: 319.5; Sigma St. Louis, Mo., USA) by measuring the growth of the parasite using SYBR green 1-based fluorescence detection (Dery et al. 2015). The in vitro drug susceptibility assay was performed for at least 3 hours after synchronization of $P$. falciparum. A $180 \mu \mathrm{L}$ of $P$. falciparum culture (triplicate for each strain) was treated with chloroquine at different concentrations. The chloroquine concentration was diluted 2fold serial reduction of its concentrations from its working stock (1000 $\mathrm{ng} / \mathrm{mL}$ ) across the 96 -well plate. There was also a well-prepared control group (without chloroquine), and only washed RBCs (background calibrator). Each well was resuspended and incubated for at least 72 hours in $37^{\circ} \mathrm{C}, \mathrm{CO}_{2}$ incubator chamber (regulated gas contains $5 \% \mathrm{CO}_{2}$ ) prior to the addition of SYBR Green I (Thermo Fisher Scientific, United Scientific) for fluorescence spectrometry analysis. Analysis using SpectraMax ${ }^{\circ}$ M5e Microplate Reader (Molecular Device) with excitation and emission wavelength bands cantered at 485 and $530 \mathrm{~nm}$, respectively. The fluorescence value was plotted against the chloroquine concentration and the $50 \%$ inhibitory concentration $\left(\mathrm{IC}_{50}\right)$ was determined by using dose-response curve by non-linear regression analysis using PRISM Version 8.0.2 as described by Le Nagard and Kaddouri (Kaddouri et al, 2006) .

\section{DNA extraction}

The extraction of genomic DNA was performed by QIAamp DNA Blood Mini Extraction Kit (Qiagen, Hilden, Germany) following the manufacturer's protocol. DNA extraction was carried out during the trophozoite-predominant developmental stage for both strains. The DNA concentration was measured using NanoDrop ${ }^{\mathrm{Ts}} 1000$ Spectrophotometer (Thermo Fisher Scientific, Waltham, Massachusetts, United States) and the DNA concentration was recorded using Nanodrop 2000 Spectrophotometer (Thermo Fisher Scientific, United States) with quality ranges 1.8 to 2.0 under absorbance 260/280 (Abs260/280).

\section{SNPs determination on Pfcrt and Pfmdr-1 gene}

A set of primers were designed via Vector NTi ver 9 by targeting single nucleotide polymorphisms (SNPs) at different codon sites of the gene using sequence obtained from gene bank (Pfcrt accession no: AF030694 and Pfmdr-1 accession no: S53996) as shown in Table 1 and Table 2, respectively. The conventional PCR was performed with PCR master mix contains of $9.3 \mu \mathrm{L}$ TE buffer, $4.0 \mu \mathrm{LPCR}$ reaction buffer, 1.2 $\mu \mathrm{L} \mathrm{MgCl}_{2}, 1.0 \mu \mathrm{L}$ forward primer, $1.0 \mu \mathrm{L}$ reverse primer, $0.4 \mu \mathrm{L}$ dNTPs and $0.2 \mu \mathrm{L}$ DNA polymerase. The PCR was performed using Bio-Rad MyCycler $^{\text {Tm}}$ Thermal Cycler System (Bio-rad, Hercules, California, United States) using the following programs; initial denaturation temperature of $95^{\circ} \mathrm{C}$ for 15 minutes, followed by 30 cycles of denaturation, annealing and extension at $95^{\circ} \mathrm{C}$ for 30 seconds, $48-72^{\circ} \mathrm{C}$ for 45 seconds and $72^{\circ} \mathrm{C}$ for 60 seconds per $\mathrm{kb}$ and final extension at $72^{\circ} \mathrm{C}$ for 7 minutes. All the PCR products were subjected for purification before sent for sequencing using QIAEX II Gel Extraction Kit (Qiagen, Germany) by following the manufacturer's protocol. The sequencing results were analysed using Bioedit software (version 7.2 .5 ) by aligning the sequences for both parasite strain (3D7 and W2 strain) with gene bank (Pfcrt; AF030694 and Pfmdr-1; S53996).

\section{Ethics approval}

Ethical clearance for the study was granted by the Universiti Sains Malaysia, Human Research Ethics Committee with approval number USM/JEPEM/1500049. Informed consent was obtained from all participants, or their parents or legal guardians in case of children and adolescents. 
Table 1: List of primer for detection of specific SNPs occurs at position 72, 73, 74, 76, 219, 220 , 326,356 and 371 on the $P f$ crt gene

\begin{tabular}{|c|c|c|c|}
\hline Point mutation & Primer sequence & Product size (bp) & $\begin{array}{l}\text { Position in genomic } \\
\text { (AF030694) }\end{array}$ \\
\hline $72,73,74,76$ & $\begin{array}{l}\text { Pfcrt76F }=\text { TGT GCT CAT GTG TTT AAA CTT } \\
\text { Pfcrt76R }=\text { CAA AAC TAT AGT TAC CAA TTT }\end{array}$ & 145 & $\begin{array}{l}23786-23800 \\
23908-23930\end{array}$ \\
\hline 219,220 & $\begin{array}{l}\text { Pfcrt219F }=\text { CTC GGA GCA GTT ATT ATT GT } \\
\text { Pfcrt219R }=\text { ATG TTT GAA AAG CAT ACA GG }\end{array}$ & 295 & $\begin{array}{l}24541-24560 \\
24816-24835\end{array}$ \\
\hline 271 & $\begin{array}{l}\text { Pfcrt271F = ATT GTT CAC TTC TTG TCT TA } \\
\text { Pfcrt271R = GAA AAT CCT ATT TTA CCT CT }\end{array}$ & 260 & $\begin{array}{l}25031-25050 \\
25271-25290\end{array}$ \\
\hline 326 & $\begin{array}{l}\text { Pfcrt326F = ACG GAG CAT GGG TAA GAA GC } \\
\text { Pfcrt326R }=\text { CCC ATA TTT ATT TCC TCT TG }\end{array}$ & 260 & $\begin{array}{l}25441-25460 \\
25681-25700\end{array}$ \\
\hline 356,371 & $\begin{array}{l}\text { Pfcrt356F }=\text { TTT CTA CCA TGA CAT ATA C } \\
\text { Pfcrt356R }=\text { CCA AAG TTA CGA AAT CTA AT }\end{array}$ & 319 & $\begin{array}{l}25801-25819 \\
26101-26120\end{array}$ \\
\hline
\end{tabular}

Table 2: List of primers for detection of specific SNPs occurs on the Pfmdr1 gene

\begin{tabular}{|c|c|c|c|}
\hline Point mutation & Primer sequence & $\begin{array}{c}\text { Product } \\
\text { size } \\
\text { (bp) }\end{array}$ & $\begin{array}{l}\text { Position in genomic } \\
\text { (S53996) }\end{array}$ \\
\hline 86,184 & $\begin{array}{l}\text { Pfmdr86F = TAC CTG CAC AAC ATA GAA AAA TTA TT } \\
\text { Pfmdr86R = TAA AGC CTC TTC TAT AAT GGA CAT }\end{array}$ & 569 & $\begin{array}{l}146-170 \\
691-714\end{array}$ \\
\hline 1034,1042 & $\begin{array}{l}\text { Pfmdr1034F }=\text { TGT AAT TTG ATA GAA AAA GCT ATT G } \\
\text { Pfmdr1034R }=\text { TAA ATA AGG ATT TCA TAA AGT CAT C }\end{array}$ & 191 & $\begin{array}{l}3016-3040 \\
3181-3206\end{array}$ \\
\hline 1246 & $\begin{array}{l}\text { Pfmdr1246F }=\text { AAT GAA TTT TCA AAC CAA TCT GGA T } \\
\text { Pfmdr1246R = TTG GTA ATG ATT CGA TAA ATT CAT C }\end{array}$ & 266 & $\begin{array}{l}3631-3656 \\
3871-3896\end{array}$ \\
\hline
\end{tabular}

\section{RESULTS}

Investigation of chloroquine susceptibility was determined by establishment dose-response of two $P$. falciparum lab-adapted strain (3D7; chloroquine sensitive and W2; chloroquine resistant) as shown in Figure 1. The curve shows that the $\mathrm{IC}_{50}$ obtained for $3 \mathrm{D} 7$ is $32.98 \mathrm{nM}$ $(10.55 \mathrm{ng} / \mathrm{mL})$ whilst for W2 is $691.21 \mathrm{nM}(221.10 \mathrm{ng} / \mathrm{mL})$. Meanwhile, the SNPs analysis shows the amino acid sequence of PfCRT at positions $72,73,74,75$ and 76 of 3D7 strain is CVMNK and CVIET for W2 strain (Table 3). The alignment result for PfMDR-1 gene shows the amino acid sequence at positions $83,84,85,86$ and 87 of $3 \mathrm{D} 7$ strain is KNMNL while for W2 strain is KNMYL (Table 4).

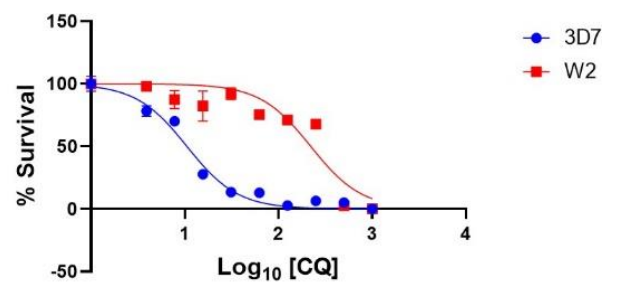

Figure 1: Dose-response curve of survival (\%) against $\log _{10}[\mathrm{CQ}]$ chloroquine susceptibility for $P$. falciparum $3 \mathrm{D} 7$ and W2 strain

\section{DISCUSSION AND CONCLUSION}

Continuous exposure of parasite cultures to a chemotherapeutic agent has contributed to the development of drug resistant parasite in the lab. However, the possibility that the susceptibility changes developed in the laboratory after long cryopreservation of the parasites has never been reported. Thus, this study is aimed to investigate the susceptibility of two common lab-adapted $P$. falciparum strains (chloroquine sensitive;3D7 and chloroquine resistant; W2) after 16 years preservation in liquid nitrogen towards chloroquine. Based on Khairul's group study backdated in 2006-2007 showed that chloroquine IC $_{50}$ of $3 \mathrm{D} 7$ was $13.84 \mathrm{nM}$ (Khairul et al, 2006)) and W2 was $208.27 \mathrm{nM}$ (Min et $a l$, 2007)). Meanwhile, this recent study shows that the susceptibility of the chloroquine concentration is 3 -fold higher of both strains when compared to the $\mathrm{IC}_{50}$ before cryopreservation. However, the mechanism or how this happen is not fully understand. One of possible reason is that due to the method applied for the susceptibility test. It happens that last 16 years ago the susceptibility assay was done using hypoxanthine technique where this study using fluorescence-based technique which is reported to be more sensitive.

Radioactively labelled hypoxanthine $\left(3 \mathrm{H}^{+}\right.$-Hypoxanthine) uptake assay was once replaced by fluorescence dye method, as the former assay is cumbersome, expensive and often limited to well-sourced radioactive laboratories and equipment. There are several fluorescence dyes that can be used to stain the DNA, such as DAPI, PicoGreen and SYBR Green I. SBYR Green I was selected for this study due to its low signal-to-noise ratio of 3:1 if compared to DAPI which is 9:1 (Baniecki et al., 2007). SYBR Green I is the preferred dye because it is convenient, relatively rapid, reproducible and less costly than radioisotope (Dennull et al., 2009). The dye has gained popularity it also reduced some hurdles associated with in vitro $P$. falciparum drug susceptibility assay.

Other than that, it is found that prolonged freezing of the parasite might give a stress environment which probably cause changes on the genetic material. Thus, our group tried to investigate this hypothesis by analysing single nucleotide polymorphism (SNP) to two important genes associated with chloroquine resistance in $P$. falciparum which is Plasmodium falciparum chloroquine resistance transporter protein (PfCRT) and Plasmodium falciparum multiple drug resistance 1 protein (PfMDR-1) that encoded by the PfCRT and PfMDR-1 gene respectively. Moers suggested that $P f C R T$ and $P f M D R-1$ genes played an important role in the development of chloroquine-resistance (Moers et al., 2015). Besides, Vathsala claimed that PfMDR-1 that encoded the pglycoprotein was initially proposed as a determinant of the chloroquineresistance phenotype (Vathsala et al., 2004). These proteins can be found 
at the food vacuole membrane of $P$. falciparum.

From the alignment result, we indicate that CQ resistant $P$. falciparum Dd2 strain encoded an amino acid pattern CIETSETI at positions 72, 74, 75, 76, 219, 220, 271, 356 and 371 on Pfcrt gene and CMNKAQIR respectively in 3D7, CQ-sensitive $P$. falciparum strain. Meanwhile, FYSND for resistance and NYSND for sensitive strain at positions 86, 184, 1034, 1042 and 1246 on Pfmdr1. Our Pfcrt results are consistently similar as reported by other studies. However, Shilea A. Peel, reported that the putative mutation at codon position 86 of W2 in Pfmdr1, referred to Thailand strain $\mathrm{K} 1$, which resulting in asparagines (AAT) to tyrosine (TAT) changes at amino acid 86 which can cause the W2 strain resistant to other drug such Mefloquine (Mef) (Peel et al., 1994).

Even though our finding shows that the level of chloroquine susceptibility increases to 3 -fold for each strain after being revived from cryopreservation, but the SNPs results reveal no changes at gene level of two proteins associated with the chloroquine resistant and our SNP finding is consistent with other studies (Antony et al., 2016, Cui et al., 2015).

Table 3: SNPs analysis of Pfcrt gene

\begin{tabular}{cccc}
\hline $\begin{array}{c}\text { Codon } \\
\text { position }\end{array}$ & $\begin{array}{c}\text { AFO30694 } \\
\text { (resistant type) }\end{array}$ & \multicolumn{2}{c}{ Sequencing Results } \\
\hline & & W2 & 3D7 \\
\hline 72 & TGT (Cys) & TGT & TGT \\
74 & ATT (Ile) & ATT & ATG (Met) \\
75 & GAA (Glu) & GAA & AAT (Asn) \\
76 & ACA (Thr) & ACA & AAA (Lys) \\
219 & AGT (Ser) & AGT & AGT (Ser) \\
220 & TCC (Ser) & TCC & GCC (Ala) \\
271 & GAA (Glu) & GAA & CAA (Gln) \\
326 & AGC (Ser) & ND & ND \\
356 & ACA (Thr) & ACA & ATA (Ile) \\
371 & ATA (IIe) & ATA & AGA (Arg) \\
\hline
\end{tabular}

ND; not detected, Cys; Cysteine, Ile; Isoleucine, Glu; Glutamic acid,

Thr; Threonine, Ser; Serine, Met; Methionine, Asn; Asparagine,

Lys; Lysine, Ala; Alanine, Gln; Glutamine, Arg; Arginine

Table 4: SNPs analysis of Pfmdr-1 gene

\begin{tabular}{cccc}
\hline $\begin{array}{c}\text { Codon } \\
\text { position }\end{array}$ & $\begin{array}{c}\text { S53996 } \\
\text { (resistant type) }\end{array}$ & \multicolumn{2}{c}{ Sequencing Results } \\
\hline & & W2 & 3D7 \\
\hline 86 & TAT (Tyr) & TAT & AAT (Asn) \\
184 & TTT (Phe) & TAT & TAT (Tyr) \\
1034 & TGT Cys) & AGT & AGT (Ser) \\
1042 & GAT (Asp) & AAT & AAT (Asn) \\
1246 & TAT (Tyr) & GAT & GAT (Asp) \\
\hline \multicolumn{2}{c}{ Tyr; Tyrosine, Phe; Phenylalanine, Cys; Cysteine, Asp; Aspartic acid, }
\end{tabular}

Asn; Asparagine, Ser; Serine, Asp; Aspartate

\section{DISCLOSURES}

All authors declare that there is no conflict of interest regarding the publication of this article.

\section{ACKNOWLEDGEMENT}

Immeasurable appreciation and deepest gratitude are extended by the authors to individuals involved in all kind of contribution to this research particularly to the Research University Individual Grant Scheme from Universiti Sains Malaysia (Grant no: 1001/CIPPM/812150) and Malaysia, Ministry of Education under the Higher Institute Centre of Excellent (HICoE) program (Grant No: 311/CIPPM/4401005) for the financial support. We also would like to thank the Institute for Research in Molecular Medicine (INFORMM) and School of Health Sciences (PPSK), Universiti Sains Malaysia for the permission to use the malaria laboratory and biomedical facilities respectively. Lastly, we would like to thank BEI Resources, NIAID, NIH for the two parasites used in this study: Plasmodium falciparum strain W2, MRA-157, contributed by Dennis E. Kyle and Plasmodium falciparum strain 3D7, MRA-102, contributed by Daniel J. Carucci.

\section{REFERENCES}

Antony, H. A., Das, S., Parija, S. C. \& Padhi, S. (2016). Sequence analysis of pfcrt and pfmdrl genes and its association with chloroquine resistance in Southeast Indian Plasmodium falciparum isolates. Genomics Data, 8, 85-90. https://doi.org/10.1016/j.gdata.2016.04.010

Baniecki, M. L., Wirth, D. F. \& Clardy, J. (2007). High-throughput Plasmodium falciparum growth assay for malaria drug discovery. Antimicrobial Agents and Chemotherapy, 51(2), 716-723 https://doi.org/10.1128/AAC.01144-06

Basco, L. K., \& Ringwald, P. (2001). Analysis of the key pfcrt point mutation and in vitro and in vivo response to chloroquine in Yaounde, Cameroon. The Journal of infectious diseases, 183(12), 1828-1831. https://doi.org/10.1086/320726

Butcher, G. A., \& Cohen, S. (1971). Short-term culture of Plasmodium knowlesi. Parasitology, 62(2), 309-320. https://doi.org/10.1017/S0031182000071547

Cheruiyot, J., Ingasia, L.A., Omondi, A.A., Juma, D.W., Opot, B.H., Ndegwa, J.M., Mativo, J., Cheruiyot, A.C., Yeda, R., Okudo, C. and Muiruri, P. 2014 Polymorphisms in Pfmdrl, Pfcrt, and Pfnhel genes are associated with reduced in vitro activities of quinine in Plasmodium falciparum isolates from western Kenya. Antimicrobial Agents and Chemotherapy, 58(7), 3737-3743 https://doi.org/10.1128/AAC.02472-14

Cui, L., Mharakurwa, S., Ndiaye, D., Rathod, P. K. \& Rosenthal, P. J. (2015a) Antimalarial Drug Resistance: Literature Review and Activities and Findings of the ICEMR Network. The American Journal of Tropical Medicine and Hygiene, 93(3 Suppliment), 57-68. https://doi.org/10.4269/ajtmh.15-0007

Dennull, R. A., Reinbold, D. D., Waters, N. C. \& Johnson, J. D. (2009). Assessment of malaria in vitro drug combination screening and mixed-strain infections using the malaria Sybr green I-based fluorescence assay. Antimicrobial Agents and Chemotherapy, 53(6), 2557-2563. https://doi.org/10.1128/AAC.01370-08

Dery, V., Duah, N. O., Ayanful-Torgby, R., Matrevi, S. A., Anto, F., \& Quashie, N. B. (2015). An improved SYBR Green-1-based fluorescence method for the routine monitoring of Plasmodium falciparum resistance to anti-malarial drugs. Malaria journal, 14(1), 1-6. https://doi.org/10.1186/s12936-015-1011-x

Fidock, D. A., Nomura, T., Talley, A. K., Cooper, R. A., Dzekunov, S. M., Ferdig, M. T., ... \& Wootton, J. C. (2000). Mutations in the P. falciparum digestive vacuole transmembrane protein PfCRT and evidence for their role in chloroquine resistance. Molecular cell, 6(4), 861-871. https://doi.org/10.1016/S1097-2765(05)00077-8

Kaddouri, H., Nakache, S., Houzé, S., Mentré, F., \& Le Bras, J. (2006). Assessment of the drug susceptibility of Plasmodium falciparum clinical isolates from Africa by using a Plasmodium lactate dehydrogenase immunodetection assay and an inhibitory maximum effect model for precise measurement of the 50percent inhibitory concentration. Antimicrobial agents and chemotherapy, 50(10), 3343-3349. https://doi.org/10.1128/AAC.00367-06

Khairul, M. F. M., Min, T. H., Low, J. H., Nasriyyah, C. H. C., A shikin, A. N., Norazmi, M. N., ... \& Raju, S. S. (2006). Fluoxetine potentiates chloroquine and mefloquine effect on multidrug-resistant Plasmodium falciparum in vitro. Japanese journal of infectious diseases, 59(5), 329.

Lambros, C. \& Vanderberg, J. P. (1979). Synchronization of Plasmodium falciparum erythrocytic stages in culture. The Journal of Parasitology, 418420. https://doi.org/10.2307/3280287

Li, J., Chen, J., Xie, D., Eyi, U. M., Matesa, R. A., Obono, M. M. O., Ehapo, C. S., Yang, L., Yang, H., Lin, M., Wu, W., Wu, K., Li, S. \& Chen, Z. (2015). Molecular mutation profile of Pfcrt and Pfmdr1 in Plasmodium falciparum isolates from Bioko Island, Equatorial Guinea. Infection, Genetics and Evolution, 36, 552-556.

https://doi.org/10.1016/j.meegid.2015.08.039

Mehlotra, R. K., Howes, R. E., Rakotomanga, T. A., Ramiranirina, B., Ramboarina, S., Franchard, T., ... \& Grimberg, B. T. (2017). Long-term in vitro culture of Plasmodium vivax isolates from Madagascar maintained in Saimiri boliviensis blood. Malaria journal, 16(1), 1-13. https://doi.org/10.1186/s12936-017-2090-7

Memorandum, W. H. O. (1981). Malaria parasite strain characterization, cryopreservation, and banking of isolates. Bull World Health Organ, 59, 537-548. 
Min, T. H., Khairul, M. F. M., Low, J. H., Nasriyyah, C. C., A'shikin, A. N., Norazmi, M. N., ... \& Raju, S. S. (2007). Roxithromycin potentiates the effects of chloroquine and mefloquine on multidrug-resistant Plasmodium falciparum in vitro. Experimental parasitology, 115(4), 387-392. https://doi.org/10.1016/j.exppara.2006.10.004

Moers, A. P., Hallett, R. L., Burrow, R., Schallig, H. D., Sutherland, C. J. \& van Amerongen, A. (2015). Detection of single-nucleotide polymorphisms in Plasmodium falciparum by PCR primer extension and lateral flow immunoassay. Antimicrobial Agents and Chemotherapy, 59(1), 365-371. https://doi.org/10.1128/AAC.03395-14

Mullis, K. B. (1987). U.S. Patent No. 4,683,202. Washington, DC: U.S. Patent and Trademark Office.

Njokah, M.J., Kang'ethe, J.N., Kinyua, J., Kariuki, D. and Kimani, F.T. 2016. In vitro selection of Plasmodium falciparum Pfcrt and Pfmdr1 variants by artemisinin. Malaria Journal, 15(1), 381. https://doi.org/10.1186/s12936-016-1443-y

Perlmann, P. \& Troye-Blomberg, M. (2000). Malaria blood-stage infection and its control by the immune system. Folia biologica, 46(6), 210-218.

Picot, S., Olliaro, P., de Monbrison, F., Bienvenu, A. L., Price, R. N. \& Ringwald, P. (2009). A systematic review and meta-analysis of evidence for correlation between molecular markers of parasite resistance and treatment outcome in falciparum malaria. Malaria Journal, 8, 89 . https://doi.org/10.1186/1475-2875-8-89

Reed, M. B., Saliba, K. J., Caruana, S. R., Kirk, K., \& Cowman, A. F. (2000). Pgh1 modulates sensitivity and resistance to multiple antimalarials in Plasmodium falciparum. Nature, 403(6772), 906-909. https://doi.org/10.1038/35002615

Ross, L. S., Dhingra, S. K., Mok, S., Yeo, T., Wicht, K. J., Kumpornsin, K., TakalaHarrison, S., Witkowski, B., Fairhurst, R. M., Ariey, F., Menard, D. \& Fidock, D. A. (2018). Emerging Southeast Asian PfCRT mutations confer Plasmodium falciparum resistance to the first-line antimalarial piperaquine. Nature Communications, 9(1), 3314. https://doi.org/10.1038/s41467-018-05652-0

Sanchez, C. P., Stein, W. D. \& Lanzer, M. (2007). Is PfCRT a channel or a carrier? Two competing models explaining chloroquine resistance in Plasmodium falciparum. Trends in Parasitology, 23(7), 332-339. https://doi.org/10.1016/i.pt.2007.04.013

Sabbatani, S., Fiorino, S. \& Manfredi, R. (2010). The emerging of the fifth malaria parasite (Plasmodium knowlesi). A public health concern? The Brazilian Journal of Infectious Diseases, 14(3), 299-309. https://doi.org/10.1016/S1413-8670(10)70062-3

Schuster, F.L., 2002. Cultivation of Plasmodium spp. Clinical microbiology reviews, 15(3), pp.355-364. https://doi.org/10.1128/CMR.15.3.355-364.2002

Sharma, A. \& Khanduri, U. (2009). How benign is benign tertian malaria? Journal of Vector Borne Diseases, 46(2), 141.

Sidhu, A. B. S., Verdier-Pinard, D., \& Fidock, D. A. (2002). Chloroquine resistance in Plasmodium falciparum malaria parasites conferred by pfcrt mutations. Science, 298(5591), 210-213. https://doi.org/10.1126/science.1074045

Siswantoro, H., Russell, B., Ratcliff, A., Prasetyorini, B., Chalfein, F., Marfurt, J., Kenangalem, E., Wuwung, M., Piera, K. \& Ebsworth, E. (2011). In vivo and in vitro efficacy of chloroquine against Plasmodium malariae and P. ovale in Papua, Indonesia. Antimicrobial Agents and Chemotherapy, 55(1), 197-202. https://doi.org/10.1128/AAC.01122-10

Sutherland, C. J., Tanomsing, N., Nolder, D., Oguike, M., Jennison, C., Pukrittayakamee, S., Dolecek, C., Hien, T. T., Do Rosário, V. E. \& Arez, A. P. (2010). Two nonrecombining sympatric forms of the human malaria parasite Plasmodium ovale occur globally. The Journal of Infectious Diseases, 201(10), 1544-1550. https://doi.org/10.1086/652240

Tang, T.-H. T., Salas, A., Ali-Tammam, M., del Carmen Martínez, M., Lanza, M., Arroyo, E. \& Rubio, J. M. (2010). First case of detection of Plasmodium knowlesi in Spain by Real Time PCR in a traveller from Southeast Asia. Malaria Journal, 9(1), 219. https://doi.org/10.1186/1475-2875-9-219

Thomas, S. M., Ndir, O., Dieng, T., Mboup, S., Wypij, D., Maguire, J. H. \& Wirth, D. F. (2002). In vitro chloroquine susceptibility and PCR analysis of pfcrt and PfMDR-1 polymorphisms in Plasmodium falciparum isolates from Senegal. The American Journal of Tropical Medicine and Hygiene, 66(5), 474-480. https://doi.org/10.4269/ajtmh.2002.66.474

Valderramos, S. G. \& Fidock, D. A. (2006). Transporters involved in resistance to antimalarial drugs. Trends in Pharmacological Sciences, 27(11), 594-601. https://doi.org/10.1016/j.tips.2006.09.005

Vathsala, P., Pramanik, A., Dhanasekaran, S., Devi, C. U., Pillai, C., Subbarao, S., Ghosh, S., Tiwari, S., Sathyanarayan, T. \& Deshpande, P. (2004). Widespread occurrence of the Plasmodium falciparum chloroquine resistance transporter (Pfcrt) gene haplotype SVMNT in P. falciparum malaria in India. The
American Journal of Tropical Medicine and Hygiene, 70(3), 256-259. https://doi.org/10.4269/ajtmh.2004.70.256

Wellems, T. E. \& Plowe, C. V. (2001). Chloroquine-resistant malaria. The Journal of Infectious Diseases, 184(6), 770-776. https://doi.org/10.1086/322858

World Health Organization. (2019). World Malaria Report 2018, World Heath Organization. Available at: https://apps.who.int/iris/handle/10665/275867.

Citation:

Pua, J. Y., Abu Bakar, N., Nik Kamarudin, N. A. A. ., Ghazali, S. Z. ., \& Khairul Mohd Fadzli Mustaffa. (2020). Long cryopreserved lab-adapted Plasmodium falciparum increases resistance to chloroquine but not its susceptibility [Provisional Acceptance]. Life Sciences, Medicine and Biomedicine, 4(9). https://doi.org/10.28916/lsmb.4.9.2020.66

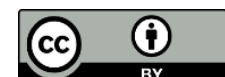

Copyright (C) 2020 by the Author(s). Life Sciences, Medicine and Biomedicine (ISSN: 2600-7207) Published by Biome Journals - Biome Scientia Sdn Bhd. Attribution 4.0 International (CC BY 4.0). This open access article is distributed based on the terms and conditions of the Creative Commons Attribution license https://creativecommons.org/licenses/by/4.0/

Life Sciences, Medicine and Biomedicine ISSN: 2600-7207 